Research article

\title{
Neural signature of tDCS, tPCS and their combination: Comparing the effects on neural plasticity
}

\author{
Aurore Thibaut (PhD) $)^{\mathrm{a}, \mathrm{b}}$, Cristina Russo $(\mathrm{MSc})^{\mathrm{a}, \mathrm{c}}$, Leon Morales-Quezada (MD, PhD) ${ }^{\mathrm{a}}$, \\ Aura Hurtado-Puerto (MD)d ${ }^{\mathrm{d}}$, Alícia Deitos (MSc) ${ }^{\mathrm{a}, \mathrm{e}, \mathrm{f}}$, Steven Freedman (MD, PhD) ${ }^{\mathrm{g}}$, \\ Sandra Carvalho (PhD) ${ }^{\mathrm{a}, \mathrm{h}}$, Felipe Fregni (MD, PhD, MPH, MMSc) ${ }^{\mathrm{a}, *}$ \\ a Spaulding-Labuschagne Neuromodulation Center, Spaulding Rehabilitation Hospital, Department of Physical Medicine and Rehabilitation, Harvard \\ Medical School, Boston, MA, USA \\ ${ }^{\mathrm{b}}$ Coma Science Group, GIGA-Research, University and University Hospital of Liege, Liege, Belgium \\ ${ }^{\mathrm{c}}$ Department of Psychology and Milan Center for Neuroscience-NeuroMi, University of Milano-Bicocca, Milano, Italy \\ d Laboratory for Neuropsychiatry and Neuromodulation, Transcranial Magnetic Stimulation Clinical Service, Department of Psychiatry, Massachusetts \\ General Hospital, Boston, USA \\ e Post-graduate Program in Medical Sciences, School of Medicine, Universidade Federal do Rio Grande do Sul (UFRGS), Porto Alegre, Brazil \\ ${ }^{\mathrm{f}}$ Laboratory of Pain and Neuromodulation at UFRGS, Porto Alegre, Brazil \\ ${ }^{g}$ Division of Translational Research, Beth Israel Deaconess Medical Center, Boston, MA, USA

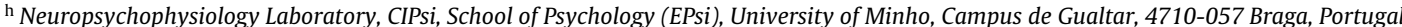

\section{H I G H L I G H T S}

- Does the combination of different NIBS techniques influence brain activity?

- tPCS increases power in alpha bandwidth by influencing bottom-up connectivity.

- tDCS has an impact on beta activity through top-down pathways.

- Combining tDCS and tPCS, applied simultaneously, decreases cortical excitability.

\section{A R T I C L E I N F O}

\section{Article history:}

Received 24 August 2016

Received in revised form 5 October 2016

Accepted 17 October 2016

Available online 17 October 2016

\section{Keywords:}

Non-invasive brain stimulation

tPCS

tDCS

EEG

Top-down/bottom-up connectivity

\begin{abstract}
A B S T R A C T
Transcranial pulsed current stimulation (tPCS) and transcranial direct current stimulation (tDCS) are two noninvasive neuromodulatory brain stimulation techniques whose effects on human brain and behavior have been studied individually. In the present study we aimed to quantify the effects of tDCS and tPCS, individually and in combination, on cortical activity, sensitivity and pain-related assessments in healthy individuals in order to understand their neurophysiological mechanisms and potential applications in clinical populations. A total of 48 healthy individuals participated in this randomized double blind sham controlled study. Participants were randomized to receive a single stimulation session of either: active or sham tPCS and active or sham tDCS. Quantitative electroencephalography (qEEG), sensitivity and pain assessments were used before and after each stimulation session. We observed that tPCS had a higher effect on power, as compared to tDCS, in several bandwidths on various cortical regions: the theta band in the parietal region $(p=0.021)$, the alpha band in the temporal $(p=0.009)$, parietal $(p=0.0063)$, and occipital $(p<0.0001)$ regions. We found that the combination of tPCS and tDCS significantly decreased power in the low beta bandwidth of the frontal $(\mathrm{p}=0.0006)$, central $(\mathrm{p}=0.0001)$, and occipital $(\mathrm{p}=0.0003)$ regions, when compared to sham stimulation. Additionally, tDCS significantly increased power in high beta over the temporal $(\mathrm{p}=0.0015)$ and parietal $(\mathrm{p}=0.0007)$ regions, as compared to sham. We found no effect on sensitivity or pain-related assessments. We concluded that tPCS and tDCS have different neurophysiological mechanisms, elicit distinct signatures, and that the combination of the two leads to no effect or a decrease on qEEG power. Further studies are required to examine the effects of these techniques on clinical populations in which EEG signatures have been found altered.
\end{abstract}

(c) 2016 Published by Elsevier Ireland Ltd.

\footnotetext{
* Corresponding author at: Spaulding-Labuschagne Neuromodulation Center, 79/96 13th Street, Charlestown, MA 02129, USA.

E-mail address: felipe.fregni@mgh.harvard.edu (F. Fregni).
} 


\section{Introduction}

One important recent insight in the field of non-invasive brain stimulation (NIBS) is that each technique has a distinct neurophysiological signature [1]. A deeper understanding of the differences between techniques of transcranial stimulation becomes critical, given the increase in the number of options and novel methods being developed. Nevertheless, few trials have compared these techniques head-to-head, particularly while using neurophysiological markers to further understand their impact on brain activity. In this study, we compared the effects of transcranial Direct Current Stimulation (tDCS; i.e., direct constant current) and Pulsed Current Stimulation (tPCS; i.e., biphasic pulsed alternating current) on brain activity, as well as on sensitivity and pain assessments.

Numerous studies on tDCS showed the efficacy of this NIBS technique to modulate cortical activity in both healthy and clinical conditions [2]. From a neurophysiological perspective, anodal tDCS increases neuronal excitability by facilitating action potentials and by modifying NMDA receptor excitability [3]. Moreover, tDCS may strengthen task-related dynamic synaptic connections [4]. This technique is widely used to modulate brain activity in clinical scenarios such as stroke rehabilitation [5], pain [6], depression [7] and tinnitus [8]. In healthy subjects, tDCS has shown to modulate pain threshold [9], pain perception and empathy [10].

Another method of NIBS, transcranial Pulsed Current Stimulation (tPCS; i.e., biphasic pulsed alternating current), has shown to enhance motor skills and cognitive function [11-13]. Preliminary evidence suggests that tPCS may induce dynamic changes in cor-

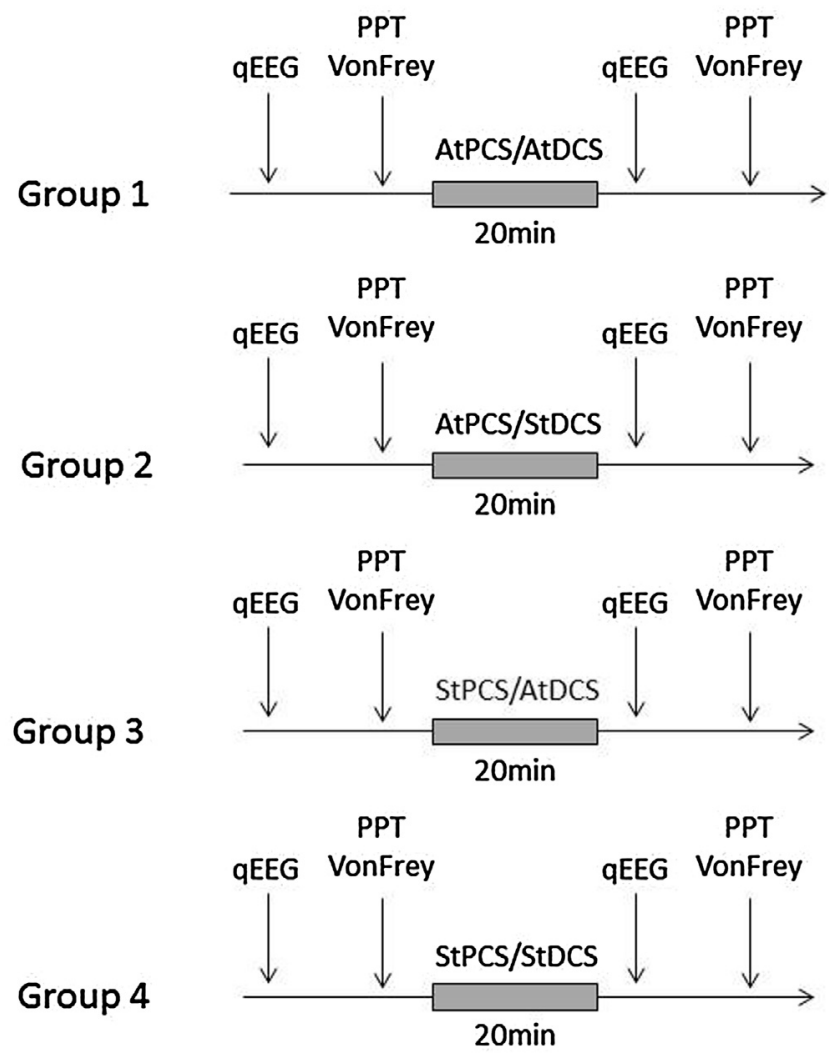

Fig. 1. Study protocol. Every participant was allocated to one of four groups: 1) active transcranial pulsed current stimulation together with active transcranial direct current stimulation (AtPCS/AtDCS); 2) active tPCS and sham tDCS (AtPCS/StDCS); 3) shan tPCS and active tDCS (StPCS/AtDCS) and 4) sham tPCS and Sham tDCS (StPCS/StDCS). Before and after each stimulation session, participants underwent a quantitative Electroencephalogram (qEEG) at rest, a Von Frey assessment and a Pain Pressure Test (PPT) before and during cold water immersion (also called conditioned pain modulation or CPM). tical areas including those responsible of cognitive processes [14]. For instance, it leads to modifications in learning processes, as a result of memory modulation, and in decision-making responses, attention and performance, by enhancing circuits [13]. In particular, recent studies suggested that tPCS applied on auricular structures can modify electrical activity of cortical and subcortical regions. Additionally, computer modeling [15] and neuropshysiological studies (using quantitative electroencephalography - QEEG) have shown that tPCS can modulate frontal [16] and interhemispheric [17] neuronal connectivity. Regarding the optimal parameters of stimulation, previous studies have shown that a current of $2 \mathrm{~mA}$ during $20 \mathrm{~min}$ [18] at a random frequency between 6 and $10 \mathrm{~Hz}$ [17] induce the most significant neurophysiological effects, especially increasing power in the alpha band, when compared to lower and higher frequencies.

Based on previous evidence, we hypothesized that tDCS would mainly change behavior $[9,19]$ through the modulation of neuronal firing under the stimulated area, whereas tPCS would modify behavioral performance through a widespread increase of cortical and subcortical connectivity [16]. Our main goal was to detect the consequences of this neuronal event through resting state EEG. Secondarily, we assessed the behavioral effects on sensitivity and pain thresholds given that a number of studies have shown that different techniques of electrical stimulation can modify these measurements. No studies directly compared tPCS with tDCS, nor the potential benefits of combining them. Therefore, in this trial, we aimed to answer the following questions: 1) Do tPCS and tDCS involve the same underlying mechanisms? 2) Does the combination of tPCS with tDCS induce a significant effect on electrophysiological measures, compared to the individual application of each technique? and finally, 3 ) Does a single session of tDCS applied without a specific task (i.e., resting state) affect cortical activity in healthy subjects?

\section{Methods}

\subsection{Study design}

This randomized, double-blind, sham-controlled trial was carried out at the Spaulding-Labuschagne Neuromodulation Center. Subjects were randomized to receive one session of either: 1) active tPCS/active tDCS; or 2) active tPCS/sham tDCS; or 3 ) sham tPCS/active tDCS, or 4) sham tPCS/sham tDCS. We used a computergenerated randomization method with blocks of four (http://www. randomization.com). Each stimulation condition was preceded and followed by an EEG recording, as well as sensitivity (i.e., Von Frey Hair assessment), and pain assessments (i.e., Prain Pressure Threshold - PPT: control condition and during cold water immersion; conditioned pain modulation - CPM) (Fig. 1).

\subsection{Participants}

Forty-eight healthy subjects (mean age, $32.4 \pm 10.6$ years; 18 females) were included in the study. Participants were eligible if they met the following criteria: age between 18 and 65; no history of neurologic, psychiatric, or unstable medical condition; no history of brain injury resulting in loss of consciousness during the last 2 years; no history of drug or alcohol abuse; no history of brain surgery or presence of metallic implants or any contraindication to tPCS or tDCS; and no current pregnancy. The study was approved by the local institutional review board and performed in accordance with the Declaration of Helsinki.

\subsection{Transcranial direct current stimulation, tDCS}

tDCS was delivered with the anode electrode positioned over the left primary motor cortex (M1-C3 based on the 10-20 inter- 
national system) and the cathode electrode over the right supra orbital region (Soterix Medical, New York, NY, USA). Stimulation lasted $20 \mathrm{~min}$ with a current of $2 \mathrm{~mA}$, with fade-in and fade-out of $30 \mathrm{~s}$. For the sham condition, stimulation parameters were the same, but the device turned off automatically after 30 s [20].

\subsection{Transcranial pulsed current stimulation, tPCS}

We used an investigational, custom-made, battery-powered, high-frequency stimulator tPCS device (Lab 8Tron AG) that delivered a quadratic biphasic alternating current using periauricular ear-clip electrodes. Stimulation parameters for tPCS were: $20 \mathrm{~min}$ of stimulation at a fixed current intensity of $2 \mathrm{~mA}$ and random noise frequency of 6-10 (as previously described - Morales-Quezada et al., 2015). The sham condition stimulation parameters were the same as those of tDCS: the device turned off automatically after $30 \mathrm{~s}$.

\subsection{Quantitative electroencephalographic recording, analysis of power and interhemispheric coherence}

We used a 64-channel, high-density Electrical Geodesic Incorporated EEG device (Electrical Geodesics, Inc., Eugene, Oregon, USA). Data was sampled at a rate of $250 \mathrm{~Hz}$, amplified and filtered using a bandpass of $0.1-70 \mathrm{~Hz}$. EEG was recorded for a total of $12 \mathrm{~min}$ ( $6 \mathrm{~min}$ eyes open and $6 \mathrm{~min}$ eyes closed). For offline analysis we used a low-pass cut filter of $40 \mathrm{~Hz}$ and a high-pass cut filter of $1 \mathrm{~Hz}$, followed by manual artifact detection and rejection by a blinded assessor.

Power and coherence were calculated using EEGLab (Delorme A., 2004) and MATLAB (MATLAB R2012a, 2000; The MathWorks Inc., Natick, Massachusetts, USA). Fast Fourier transformation (averaged windows of $5 \mathrm{~s}$ with $50 \%$ overlap) was used to calculate power $(\mu \mathrm{V} 2)$ for the following EEG bands: theta $(4-8 \mathrm{~Hz})$, alpha $(8-13 \mathrm{~Hz})$ and beta $(13-30 \mathrm{~Hz})$; and the sub-bands: low-alpha $(8-10 \mathrm{~Hz})$, high-alpha $(10-13 \mathrm{~Hz})$, low-beta $(13-20 \mathrm{~Hz})$ and high beta $(21-30 \mathrm{~Hz})$. Adjacent electrodes were selected and averaged to represent frontal, central, parietal, temporal and occipital areas.

We calculated interhemispheric coherence for these bands and sub-bands, using two different electrode pairs: E19-E56 and E14-E57, located in the frontotemporal area and including their reciprocal location in the contralateral hemisphere, as described in recent tPCS trials [16-18,21]. Welch's averaged modified periodogram method was used to find the estimated coherence of signal $\mathrm{x}$ and $\mathrm{y}$, representing each electrode site.

\subsection{Quantitative sensory assessments}

\subsubsection{Mechanical detection threshold}

To determine sensitivity threshold, we used the Von Frey Hair assessment (North Coast Medical, Inc. Morgan Hill, CA, USA), with monofilaments of $0.008-300 \mathrm{~g}$. Subjects' thresholds were assessed for perceived mechanical pressure using ascending filament gauges [22]. We applied increasingly thick monofilaments to the right thenar region until subjects, who had their eyes closed, reported the perception of the stimulus (perception threshold). This procedure was performed 3 times. The average was used to determine the sensitivity threshold.

\subsubsection{Pain pressure threshold (PPT)}

Regarding pain assessments, PPT was performed applying an increasing amount of blunt pressure using the $1-\mathrm{cm}^{2}$ hard-rubber end of an FDA-approved assessment device (Commander Algometer, JTECH Medical, Salt Lake City, UT, USA). Pressure was applied to the right thenar region with an approximate constant increase in pressure of $50 \mathrm{kPa} / \mathrm{s}$. PPT was defined as the amount of pressure with which the subject started to perceive pain. This procedure was repeated 3 times and the average value was used for analysis.

\subsubsection{Conditioned pain modulation ( $C P M)$}

For the evaluation of CPM, the same procedure applied for the PPT (test-stimulus) was repeated, this time, $30 \mathrm{~s}$ after the immersion of the left hand (up to the wrist) in cold water $\left(10-12^{\circ} \mathrm{C}\right)$. The 'conditioning stimulus' was the cold water, while the 'test-stimulus' was the localized pressure. The hand was immersed in cold water for a total of $60 \mathrm{~s}$.

\subsection{Side-effects and blinding assessments}

Immediately following administration of tDCS and tPCS, participants were asked about the occurrence of any side effects [20]. Subjects were also invited to guess whether they received active or sham tDCS/tPCS, and to rate how confident they felt about their guess in a scale of 1 (not confident at all) to 5 (completely confident).

\subsection{Sample size calculation}

As this research is exploratory in nature, the sample size of 12 subjects per group for was calculated based on the goal of testing for a difference between groups using qEEG, given that an expected difference between groups of at least $40 \%$ of increase in theta and low-alpha interhemispheric coherences.

\subsection{Statistical analyses}

For baseline characteristics, continuous variables were compared using one-way analysis of variance and categorical variables were analyzed using a $\chi 2$-test. The Kruskal-Wallis test was used to compare the effects of stimulation on power and coherence variables. Mann-Whitney tests were applied for post-hoc comparisons. The dependent variables for EEG power and coherence in each bandwidth were calculated as the difference between poststimulation and pre-stimulation.

The independent fixed variable for the power and coherence analysis was the group (group 1: active tPCS and active tDCS; group 2: active tPCS and sham tDCS; group 3: sham tPCS and active tDCS; group 4: sham tPCS and sham tDCS). Due to important movement artifacts during the 'eyes open' condition, only the 'eyes closed' condition was considered for the analyses.

Regarding sensitivity and pain assessments, we evaluated the group effect with Kruskal-Wallis test, comparing the difference (pre minus post stimulation) for Von Frey assessment, PPT and CPM.

\section{Results}

47 participants completed the study. One participant (group 1) dropped-out due to discomfort after stimulation started. EEG data from one subject (in group 3) was excluded from the analysis due to excessive artifacts in the EEG recording. Therefore, 46 subjects were included in the analysis ( $32.5 \pm 11$ years; 16 females).

\subsection{Power analysis}

There were no differences between the groups in the baseline power spectrum in the frontal, central, parietal, temporal, and occipital brain regions ( $p>0.05)$.

We found a significant group effect in the power spectrum of the theta, alpha, low alpha, low beta, and high beta bandwidths. For these bandwidths, we found differences in most of the observed regions. In the theta bandwidth, we found differences in the parietal region $(\mathrm{p}=0.034)$; in the alpha bandwidth, we found a difference in the parietal $(\mathrm{p}<0.0001)$ and occipital $(\mathrm{p}<0.0001)$ regions; in the low 
alpha bandwidth, there was a difference in the parietal $(\mathrm{p}<0.0001)$ and occipital $(\mathrm{p}<0.0001)$ regions; in the high alpha bandwidth, the difference was in the occipital region ( $\mathrm{p}=0.001)$; in the low beta bandwidth there was a difference in the frontal $(p=0.003)$, central $(p=0.043)$, temporal $(p=0.040)$, parietal $(p=0.023)$ and occipital $(\mathrm{p}=0.035)$ regions; and in the high beta bandwidth, we found a difference in the frontal ( $\mathrm{p}<0.0001)$, central $(\mathrm{p}<0.0001)$, temporal $(\mathrm{p}<0.0001)$, parietal $(\mathrm{p}<0.0001)$ and occipital $(\mathrm{p}<0.0001)$ regions. We performed the analyses per hemisphere (left and right) and did not identify any further differences.

\subsection{Post-hoc analyses}

1) Comparing tPCS versus tDCS: We identified that tPCS has a higher effect on power, as compared to tDCS, in the theta bandwidth in the parietal region $(p=0.021)$; in the alpha band in the temporal $(p=0.009)$, parietal $(p=0.0063)$ and occipital $(\mathrm{p}<0.0001)$ regions; in the low alpha band in the parietal $(\mathrm{p}=0.014)$ and occipital $(\mathrm{p}<0.0001)$ regions; and in the high alpha band in the occipital area $(\mathrm{p}=0.0001)$ (Fig. 2).

2) Comparing tPCS combined with tDCS, versus sham: We found that the combination of tPCS and tDCS significantly decreased power, as compared to sham, in the low beta bandwidth of frontal $(p=0.0006)$, central $(p=0.0001)$ and occipital $(p=0.0003)$ regions (Fig. 3$)$. There was no significant difference between TPCS combined with tDCS and sham stimulation in all the other bandwidths.

3) Comparing tDCS versus sham: The analyses showed that tDCS significantly increased power in the high beta bandwidth over temporal $(\mathrm{p}=0.0015)$ and parietal $(\mathrm{p}=0.0007)$ regions, as compared to sham (Fig. 4).

4) Comparing tPCS versus sham: We identified that tPCS significantly increased power in the alpha $(p=0.025)$ low beta $(p=0.024)$ and high beta $(p=0.0001)$ bandwidths over the temporal region; in the theta $(p=0.030)$, alpha $(p=0.0009)$, low alpha $(p=0.001)$, low beta $(p=0.013)$ and high beta $(p<0.0001)$ bandwidths over the parietal region; and in the alpha $(\mathrm{p}<0.0001)$, low alpha $(\mathrm{p}<0.0001)$ and high alpha bandwidths $(p=0.005)$ over the occipital region, as compared to sham.

Note that we did the same analyses for the left and right hemispheres separately and we did not identify any lateralization of the results

\subsection{Coherence analysis}

Regarding the interhemispheric frontal coherence, we found a significant group effect in the alpha $(\mathrm{p}=0.004)$, low alpha $(\mathrm{p}=0.034)$ and high alpha $(\mathrm{p}=0.008)$ bandwidths. The post-hoc analysis revealed a trend towards an increased interhemispheric frontal coherence in the alpha band for tPCS $(p=0.07)$.

\subsection{Pain and sensitivity assessments}

Regarding pain-related and sensitivity assessments, we did not identify any difference between groups in Von Frey $(\mathrm{p}=0.44)$, PPT $(\mathrm{p}=0.75)$ or CPM $(\mathrm{p}=0.60)$ values.

\subsection{Side-effects and blinding assessments}

Besides the participant who dropped-out due to reported discomfort with stimulation, all other subjects tolerated the procedure well and there were no significant adverse effects (Table 1). Blinding assessments revealed that 13 subjects (28.3\%) correctly guessed both interventions (sham or active tDCS and sham or active tPCS).
Of those guessed correctly, 8 subjects (61.5\%) reported to be 'almost' and 'completely' confident in their response, and 5 subjects (38.5\%) reported to be 'somewhat' or not confident at all in their response.

\section{Discussion}

This study shows that (i) tPCS, compared to tDCS, has a greater effect on cortical activity, as shown by EEG in alpha bands (low alpha and/or high alpha) over the parietal, temporal and occipital cortical regions, as well as interhemispheric frontal coherence in the alpha bandwidth; (ii) the combination of tDCS with tPCS has either no effect, or induces a decrease in power in low beta bandwidth in the frontal, central and occipital regions; and (iii) tDCS over the motor cortex improves power in high beta band in the parietal and temporal regions.

These findings suggest three important conclusions: 1 .tDCS and tPCS have different neurophysiological signatures; 2 . combining the two types of stimulation leads to no effect or to a decrease in brain excitability on healthy participants; and 3. tDCS applied at rest increases power in the beta bandwidth.

\section{1. $t D C S$ and $t P C S$ have different neurophysiological signatures}

tPCS has shown promising and reproducible results as a neuromodulatory tool, given the reported effects on coherence and EEG power spectrum [16-18]. In addition, it is a simple, painless, easy to use, portable, and safe technique. So far, various parameters, namely duration of stimulation, intensity and frequency have been investigated. In particular, it has been shown that a tPCS current of $2 \mathrm{~mA}$ during $20 \mathrm{~min}$ of a randomly pulsating frequency ranging between 6 and $10 \mathrm{~Hz}$ induced the most significant changes in brain activity. Our data confirms the results observed in previous trials with tPCS [16-18], demonstrating that this stimulation has a consistent impact on cortical activity, as measured by power and connectivity in alpha frequencies. Furthermore, our results showed that a single session of tPCS is sufficient to induce reliable cortical changes in alpha bands (global, low and high alpha), using the random frequencies parameter of $6-10 \mathrm{~Hz}$.

As a priori hypothesized, the effects of tDCS and tPCS are not mediated by the same neurophysiological mechanisms. Previous studies with resting state tDCS over the motor cortex, suggested that tDCS does not induce action potentials, but instead changes the threshold for action potential generation in the neurons [23-25]. Thus, tDCS changes the likelihood of neuronal discharge and is theoretically not able to change spontaneous brain oscillatory activity, especially that which is thought to be dependent on the summation of postsynaptic potentials, reflected in EEG. tPCS seems to influence deeper brain structures than tDCS, such as the brain stem, the thalamus and the hypothalamus, based on head model current simulation [15], whereas tDCS increases cortical excitability under the stimulating electrodes [26]. On the other hand, tPCS with a random frequency of $6-10 \mathrm{~Hz}$ seems to modulate brain oscillation and synchronize within the power spectrum within these frequencies. As compared to transcranial Alternating Current Stimulation (tACS), using sinusoidal waves, tPCS delivers randomly generated quadratic pulses, which may in turn, increase the power and connectivity of endogenous generated brain oscillation, under the mechanisms of stochastic facilitation, to synchronize cortical activity within the boundaries of the applied random frequencies. Based on these assumptions, we can hypothesize that by improving coherence and information integration, tPCS could enhance speed processing, as already shown [13], and could be useful for the treatment of neurological conditions such as traumatic brain injury or attention-deficit/hyperactivity disorder (ADHD), or any other condition in which connectivity needs to be modulated. For 


\section{tPCS versus tDCS}

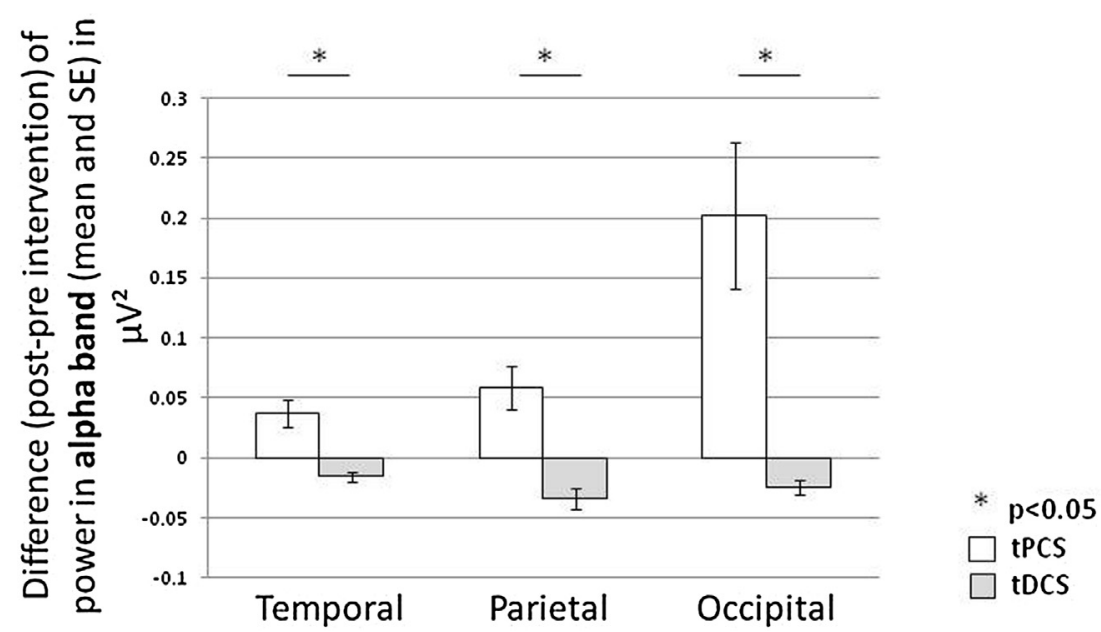

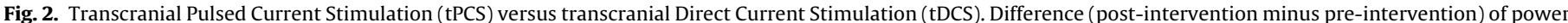
in the alpha bandwidth for active tPCS (white) versus active tDCS (light grey) in temporal, parietal and occipital regions.

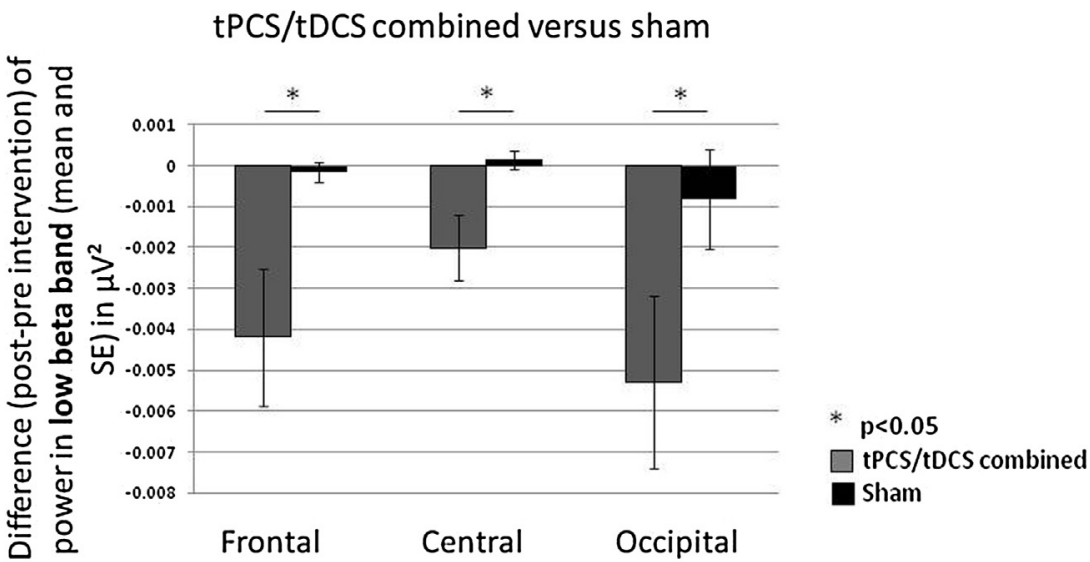

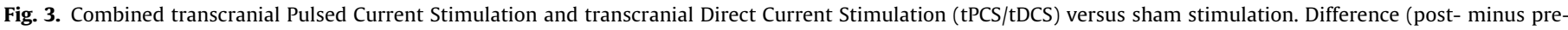
intervention) in power in the low beta bandwidth for combined tPCS/tDCS (grey) versus sham stimulation (black) in frontal, central and occipital regions.

Table 1

Side effects of tDCS and tPCS.

\begin{tabular}{|c|c|c|c|c|c|c|c|c|}
\hline & \multicolumn{2}{|c|}{ Group $1(n=11)$} & \multicolumn{2}{|c|}{ Group $2(n=12)$} & \multicolumn{2}{|c|}{ Group $3(n=12)$} & \multicolumn{2}{|c|}{ Group $4(n=12)$} \\
\hline & Active tPCS & Active tDCS & Active tPCS & Sham tDCS & Sham tPCS & Active tDCS & Sham tPCS & Sham tDCS \\
\hline Neck Pain & 0 & 0 & 0 & 0 & 0 & 0 & 0 & 0 \\
\hline Pain in area of electrodes & 0 & 2 & 1 & 0 & 1 & 0 & 1 & 0 \\
\hline Burns in area of electrodes & 0 & 3 & 1 & 2 & 1 & 2 & 3 & 0 \\
\hline Tingling & 7 & 1 & 10 & 0 & 10 & 0 & 9 & 0 \\
\hline Skin Redness & 0 & 5 & 0 & 0 & 0 & 3 & 0 & 0 \\
\hline Sleepiness & 0 & 0 & 0 & 0 & 1 & 0 & 0 & 0 \\
\hline Trouble Concentrating & 0 & 0 & 0 & 0 & 0 & 0 & 0 & 0 \\
\hline Acute Mood Change & 0 & 0 & 0 & 0 & 0 & 0 & 0 & 0 \\
\hline Itching & 0 & 0 & 0 & 0 & 1 & 0 & 0 & 0 \\
\hline Vibration & 0 & 0 & 0 & 0 & 0 & 0 & 1 & 0 \\
\hline Dizziness $^{\mathrm{a}}$ & 0 & & 2 & & 0 & & 0 & \\
\hline Headache $^{\mathrm{a}}$ & 1 & & 0 & & 0 & & 0 & \\
\hline
\end{tabular}

Number of subjects reporting the side effect.

a For headaches and dizziness the subjects could not differentiate tPCS from tDCS side-effects.

instance, developmental delay in the form of language dyspraxia has been successfully treated with tPCS as a co-adjuvant technique for speech therapy as shown by anecdotal data from our center. Particularly, an improvement in vocabulary and in performance during speech therapy sessions was observed. These improvements might reflect the effect of tPCS facilitating circuit maturation through improved coherence. In addition, since tPCS seems to have an effect on deep brain structures, potentially stimulating thalamo-cortical circuits, we can hypothesize that this technique could be beneficial 


\section{tDCS versus sham}

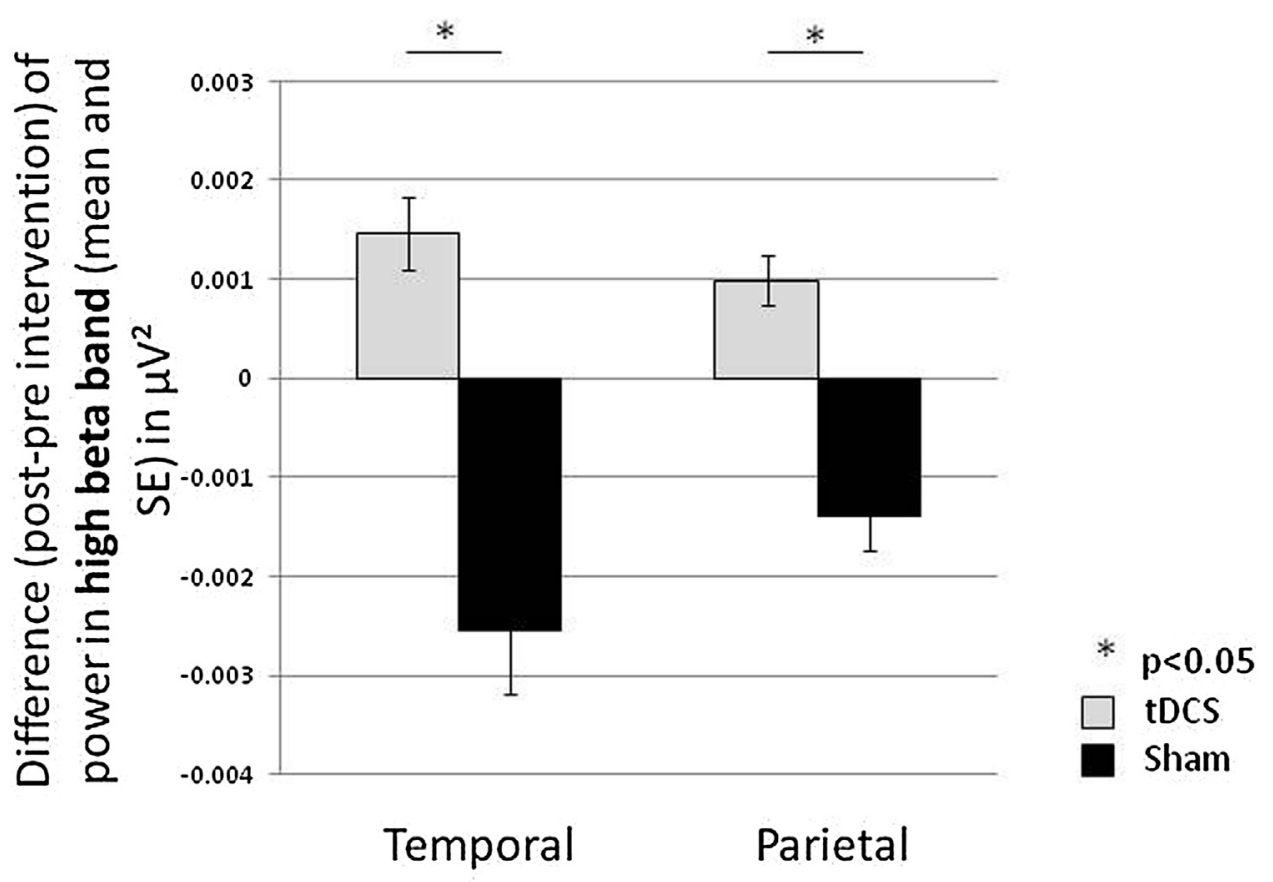

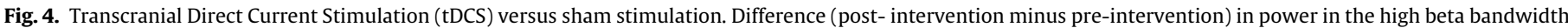
for tDCS (light grey) versus sham stimulation (black) in temporal and parietal regions.

for patients with disorders of consciousness, since these circuits are usually disturbed in this condition [27].

These are important insights, as the effects of electrical stimulation techniques are frequently considered on the basis of behavioral effects only. For instance, if tDCS and tPCS have similar attention enhancing effect, it is often mistakenly interpreted that this effect is mediated by similar neural mechanisms. Although in this study neither technique generated a behavioral change, the demonstration that they induced different neural mechanisms at rest suggests that further research should be guided by neural mechanisms rather than solely by behavioral effects. This notion would be critical to develop these techniques as potential clinical tools.

\subsection{Combining the two types of stimulation leads to no effect or to a decrease in brain excitability}

Our results highlight the negative effect of concurrently combining tDCS and tPCS in healthy participants, since both stimulations seem to cancel each other. tPCS is thought to influence deeper brain structures, such as the brainstem where it can exert an effect on the reticular formation and, consequently, modulate the signals arriving to the thalamus. Based on the previous tPCS results (see previous paragraph) it seems feasible that the thalamus, due to its role as electrographical generator through the thalamo-cortical circuits, could stimulate the bottom-up connectivity. On the contrary, tDCS seems to target a top-down cortico-thalamic pathway [28]. Thus, it may be possible that the sum of effects, as distinct as the ones induced by tDCS and tPCS, can result in an the cancellation of the individual signatures of these techniques in brain activity.

However, it is also important to stress that this cancellation of effects was observed in healthy participants and does not necessarily translate to patients with neurological conditions and disrupted cortico-thalamo-cortical loop [29-31]. In addition, it is possible that combining the two types of stimulation, not simultaneously but one after the other, could lead to the expected additive results and not the observed cancelled outcomes. As mentioned in the introduction, tDCS may increase the neuronal firing under the stimulated area and enhance learning capabilities, whereas tPCS seems to involve a more widespread cortical and subcortical network and, behaviorally, this could facilitate a task that has been previously learned [16]. While applying tDCS (before a task) and then tPCS (during a task), this could induce the expected additive effects of the two NIBS techniques.

\section{3. $t D C S$ at rest increases power in the beta bandwidth}

We showed that tDCS over the left primary motor cortex (M1) induces a beta-power enhancement after $20 \mathrm{~min}$ of stimulation. A previous study demonstrated similar results after a single stimulation session over the prefrontal cortex [32]. The authors concluded that tDCS could change the brain to a "ready state" to perform cognitive (if the prefrontal area was stimulated) or motor (if the primary motor area was stimulated) tasks.

Compared to previous studies, we did not find an effect of stimulation on pain thresholds. These results contrast with previous tDCS studies on pain in both, patients [33-35] and healthy subjects [9]. This might be explained by the timeline of our protocol, since the pain assessments were performed up to $40 \mathrm{~min}$ after the stimulation due to the time it took to prepare and record EEG. Another possible explanation could be a type II error $(\beta)$, since these measurements were secondary outcomes and our sample size was powered to detect qEEG differences. In addition, it should be noted that current literature on tDCS has shown that repetitive sessions are required in order to induce larger effects [36,37]. Therefore, a single stimulation session might not be enough to generate important clinical changes. Finally, it is more and more evident that the 
effects of $\mathrm{tDCS}$ seem to be greater when coupled with a specific task [38-40].

\section{Conclusion}

This study provides evidence of head-to-head and combined effects of a single session of tPCS and tDCS on cortical neurophysiology and pain-related assessments in healthy individuals. A single session of tPCS has greater effects on power, mainly in the alpha bandwidth, as compared to tDCS, while the combination of the two techniques can even decrease cortical activity at high frequencies. These two types of stimulation going in opposite directions (i.e., tPCS seems to stimulate bottom-up connectivity while tDCS modulates top-down pathways) could result in the elimination of their respective effects on brain activity. This observation highlights the need of careful consideration and better understanding of the underlying mechanisms of action before associating different transcranial stimulation techniques.

As it becomes clear that tPCS and tDCS have distinct physiological mechanisms and distinct effects on resting cortical activity, it would be interesting to explore these effects on clinical populations in which treatment options are limited, such as chronic pain syndromes. Indeed we have hypothesized that tPCS and tDCS have different signatures in cognitive tasks: while the first seems to improve accuracy [13], the second seems to enhance reaction time $[41,42]$. Therefore, further studies investigating the effects of tPCS and tDCS applied consecutively should be performed. This will help to determine if these two theoretically complementary neuromodulation techniques can have additive effects when they are used in a more appropriate order: e.g., first tDCS when learning a specific task, preparing the subject to perform this task; then tPCS during the task to improve performance.

\section{Conflict of interest}

The authors have no conflict of interest to declare.

\section{Acknowledgments}

This research was supported by Labuschagne Foundation. Dr. Thibaut was founded by the Belgian American Educational Foundation (BAEF) and the Duesberg Foundation. Dr. Morales-Quezada received funding support from an Institutional National Research Service Award from the National Center for Complementary and Integrative Health grant T32AT000051, the Ryoichi Sasakawa Fellowship Fund, and by the Program in Placebo Studies at Beth Israel Deaconess Medical Center. Deitos, A. was founded by the Coordination for the Improvement of Higher Education Personnel - CAPES, International Cooperation General Program - PGCI ( ${ }^{\circ}$ 023/11). Prof. Fregni has been supported by NIH RO1 grant (1R01HD08230201A1).

\section{References}

[1] A. Antal, C.S. Herrmann, Transcranial alternating current and random noise stimulation: possible mechanisms, Neural Plast. 2016 (2016) 1-12, http://dx. doi.org/10.1155/2016/3616807.

[2] M.A. Nitsche, L.G. Cohen, E.M. Wassermann, A. Priori, N. Lang, A. Antal, W. Paulus, F. Hummel, P.S. Boggio, F. Fregni, A. Pascual-Leone, Transcranial direct current stimulation: state of the art 2008, Brain Stimul. 1 (2008) 206-223, http://dx.doi.org/10.1016/j.brs.2008.06.004.

[3] C.J. Stagg, M.A. Nitsche, Physiological basis of transcranial direct current stimulation, Neuroscientist 17 (2011) 37-53, http://dx.doi.org/10.1177/ 1073858410386614.

[4] R. Polania, W. Paulus, M.A. Nitsche, Modulating cortico-striatal and thalamo-cortical functional connectivity with transcranial direct current stimulation, Hum. Brain Mapp. 33 (2012) 2499-2508, http://dx.doi.org/10. 1002/hbm.21380.
[5] J. Marquez, P. van Vliet, P. McElduff, J. Lagopoulos, M. Parsons, Transcranial direct current stimulation (tDCS): does it have merit in stroke rehabilitation? A systematic review, Int. J. Stroke 10 (2015) 306-316, http://dx.doi.org/10. 1111/ijs.12169.

[6] M.F. DosSantos, N. Ferreira, R.L. Toback, A.C. Carvalho, A.F. DaSilva, Potential mechanisms supporting the value of motor cortex stimulation to treat chronic pain syndromes, Front. Neurosci. 10 (2016), http://dx.doi.org/10. 3389/fnins.2016.00018.

[7] U. Palm, A. Hasan, W. Strube, F. Padberg, tDCS for the treatment of depression: a comprehensive review, Eur. Arch. Psychiatry Clin. Neurosci. (2016) 1-14, http://dx.doi.org/10.1007/s00406-016-0674-9.

[8] G.S. Shekhawat, C.M. Stinear, G.D. Searchfield, Modulation of perception or emotion? A scoping review of tinnitus neuromodulation using transcranial direct current stimulation, Neurorehabil. Neural Repair (2015), http://dx.doi. org/10.1177/1545968314567152, 1545968314567152.

[9] J.S. Reidler, M.E. Mendonca, M.B. Santana, X. Wang, R. Lenkinski, A.F. Motta, S. Marchand, L. Latif, F. Fregni, Effects of motor cortex modulation and descending inhibitory systems on pain thresholds in healthy subjects, J. Pain 13 (2012) 450-458, http://dx.doi.org/10.1016/j.jpain.2012.01.005.

[10] G.G. Rêgo, O.M. Lapenta, L.M. Marques, T.L. Costa, J. Leite, S. Carvalho, ÓF Gonçalves, A.R. Brunoni, F. Fregni, P.S. Boggio, Hemispheric dorsolateral prefrontal cortex lateralization in the regulation of empathy for pain, Neurosci. Lett. 594 (2015) 12-16, http://dx.doi.org/10.1016/j.neulet.2015.03. 042.

[11] C. Miniussi, J.A. Harris, M. Ruzzoli, Modelling non-invasive brain stimulation in cognitive neuroscience, Neurosci. Biobehav. Rev. 37 (2013) 1702-1712, http://dx.doi.org/10.1016/j.neubiorev.2013.06.014.

[12] L. Morales-Quezada, C. Cosmo, S. Carvalho, J. Leite, L. Castillo-Saavedra, J.R. Rozisky, F. Fregni, Cognitive effects and autonomic responses to transcranial pulsed current stimulation, Exp. Brain Res. 233 (2015) 701-709, http://dx.doi. org/10.1007/s00221-014-4147-y.

[13] L. Morales-Quezada, J. Leite, S. Carvalho, L. Castillo-Saavedra, C. Cosmo, F. Fregni, Behavioral effects of transcranial pulsed current stimulation (tPCS): speed-accuracy tradeoff in attention switching task, Neurosci. Res. 109 (2016) 48-53, http://dx.doi.org/10.1016/j.neures.2016.01.009.

[14] S. Jaberzadeh, A. Bastani, M. Zoghi, Anodal transcranial pulsed current stimulation: a novel technique to enhance corticospinal excitability, Clin. Neurophysiol. 125 (2014) 344-351, http://dx.doi.org/10.1016/j.clinph.2013. 08.025 .

[15] A. Datta, J.P. Dmochowski, B. Guleyupoglu, M. Bikson, F. Fregni, Cranial electrotherapy stimulation and transcranial pulsed current stimulation: a computer based high-resolution modeling study, Neuroimage 65 (2013) 280-287, http://dx.doi.org/10.1016/j.neuroimage.2012.09.062.

[16] L. Castillo Saavedra, L. Morales-Quezada, D. Doruk, J. Rozinsky, L. Coutinho, P. Faria, I. Perissinotti, Q.M. Wang, F. Fregni, QEEG indexed frontal connectivity effects of transcranial pulsed current stimulation (tPCS): a sham-controlled mechanistic trial, Neurosci. Lett. 577 (2014) 61-65, http://dx.doi.org/10.1016/ j.neulet.2014.06.021.

[17] L. Morales-Quezada, L. Castillo-Saavedra, C. Cosmo, D. Doruk, I. Sharaf, A. Malavera, F. Fregni, Optimal random frequency range in transcranial pulsed current stimulation indexed by quantitative electroencephalography, Neuroreport 26 (2015) 747-752, http://dx.doi.org/10.1097/WNR. 0000000000000415.

[18] L. Morales-Quezada, L.C. Saavedra, J. Rozisky, L. Hadlington, F. Fregni, Intensity-dependent effects of transcranial pulsed current stimulation on interhemispheric connectivity: a high-resolution qEEG, sham-controlled study, Neuroreport 25 (2014) 1054-1058, http://dx.doi.org/10.1097/WNR. 0000000000000228.

[19] B.W. Fenton, P.A. Palmieri, P. Boggio, J. Fanning, F. Fregni, A preliminary study of transcranial direct current stimulation for the treatment of refractory chronic pelvic pain, Brain Stimul. 2 (2009) 103-107, http://dx.doi.org/10. 1016/j.brs.2008.09.009.

[20] A.R. Brunoni, M.A. Nitsche, N. Bolognini, M. Bikson, T. Wagner, L. Merabet, D.J. Edwards, A. Valero-Cabre, A. Rotenberg, A. Pascual-Leone, R. Ferrucci, A. Priori, P.S. Boggio, F. Fregni, Clinical research with transcranial direct current stimulation (tDCS): challenges and future directions, Brain Stimul. 5 (2012) 175-195, http://dx.doi.org/10.1016/j.brs.2011.03.002.

[21] A. Vasquez, A. Malavera, D. Doruk, L. Morales-Quezada, S. Carvalho, J. Leite, F. Fregni, Duration dependent effects of transcranial pulsed current stimulation (tPCS) indexed by electroencephalography, Neuromodulation 19 (7) (2016) 679-688.

[22] R. Rolke, W. Magerl, K.A. Campbell, C. Schalber, S. Caspari, F. Birklein, R.D. Treede, Quantitative sensory testing: a comprehensive protocol for clinical trials, Eur. J. Pain 10 (2006) 77-88, http://dx.doi.org/10.1016/j.ejpain.2005.02. 003.

[23] M.A. Nitsche, W. Paulus, Excitability changes induced in the human motor cortex by weak transcranial direct current stimulation, J. Physiol. 527 (Pt 3) (2000) 633-639 http://www.ncbi.nlm.nih.gov/pubmed/10990547.

[24] M.A. Nitsche, D. Liebetanz, A. Antal, N. Lang, F. Tergau, W. Paulus, Modulation of cortical excitability by weak direct current stimulation-technical, safety and functional aspects, Suppl. Clin. Neurophysiol. 56 (2003) 255-276 http:// www.ncbi.nlm.nih.gov/pubmed/14677403.

[25] M.A. Nitsche, W. Paulus, Sustained escitability elevations induces by transcrnaial DC motor cortex stimulation in humans, Neurology 57 (2001) 1899-1901. 
[26] D. Keeser, F. Padberg, E. Reisinger, O. Pogarell, V. Kirsch, U. Palm, S. Karch, H.J. Moller, M.A. Nitsche, C. Mulert, Prefrontal direct current stimulation modulates resting EEG and event-related potentials in healthy subjects: a standardized low resolution tomography (sLORETA) study, Neuroimage 55 (2011) 644-657, http://dx.doi.org/10.1016/j.neuroimage.2010.12.004.

[27] S. Laureys, N.D. Schiff, Coma and consciousness: paradigms (re)framed by neuroimaging, Neuroimage 61 (2012) 478-491, http://dx.doi.org/10.1016/j. neuroimage.2011.12.041.

[28] L. Frase, H. Piosczyk, S. Zittel, F. Jahn, P. Selhausen, L. Krone, B. Feige, F. Mainberger, J.G. Maier, M. Kuhn, S. Klöppel, C. Normann, A. Sterr, K. Spiegelhalder, D. Riemann, M.A. Nitsche, C. Nissen, Modulation of total sleep time by transcranial direct current stimulation (tDCS), Neuropsychopharmacology (2016), http://dx.doi.org/10.1038/npp.2016.65.

[29] J.H. Hong, D.S. Bai, J.Y. Jeong, B.Y. Choi, C.H. Chang, S.H. Kim, S.H. Ahn, S.H. Jang, Injury of the spino-thalamo-cortical pathway is necessary for central post-stroke pain, Eur. Neurol. 64 (2010) 163-168, http://dx.doi.org/10.1159/ 000319040.

[30] J. Garcia-Panach, N. Lull, J.J. Lull, J. Ferri, C. Martinez, P. Sopena, M. Robles, J. Chirivella, E. Noe, A voxel-based analysis of FDG-PET in traumatic brain injury: regional metabolism and relationship between the thalamus and cortical areas, J. Neurotrauma 28 (2011) 1707-1717, http://dx.doi.org/10. 1089/neu.2011.1851.

[31] S. Bagnato, C. Boccagni, A. Sant'Angelo, A.A. Fingelkurts, A.A. Fingelkurts, G. Galardi, Emerging from an unresponsive wakefulness syndrome: brain plasticity has to cross a threshold level, Neurosci. Biobehav. Rev. 37 (2013) 2721-2736, http://dx.doi.org/10.1016/j.neubiorev.2013.09.007.

[32] M. Song, Y. Shin, K. Yun, Beta-frequency EEG activity increased during transcranial direct current stimulation, Neuroreport 25 (2014) 1433-1436, http://dx.doi.org/10.1097/WNR.0000000000000283.

[33] A.P. Brietzke, J.R. Rozisky, J.A. Dussan-Sarria, A. Deitos, G. Laste, P.F.T. Hoppe, S. Muller, I.L.S. Torres, M.R. Alvares-da-Silva, R.F.B. de Amorim, F. Fregni, W. Caumo, Neuroplastic effects of transcranial direct current stimulation on painful symptoms reduction in chronic hepatitis C: a phase II randomized, double blind, sham controlled trial, Front. Neurosci. 9 (2015) 498, http://dx. doi.org/10.3389/fnins.2015.00498.

[34] V. M.S, F. A, Reduction of chronic abdominal pain in patients with inflammatory bowel disease through transcranial direct current stimulation: a randomized controlled trial, Pain 157 (2016) 429-437,

http://www.elsevier.com/locate/painonline \nhttp://ovidsp.ovid.com/ovidweb.cgi? $\mathrm{T}=\mathrm{JS} \& \mathrm{PAGE}=$ reference $\& \mathrm{D}=$ emed $13 \& N E W S=\mathrm{N} \& A N=20160084149$.
[35] B. Vaseghi, M. Zoghi, S. Jaberzadeh, Does anodal transcranial direct current stimulation modulate sensory perception and pain? A meta-analysis study, Clin. Neurophysiol. 125 (2014) 1847-1858, http://dx.doi.org/10.1016/j.clinph. 2014.01.020.

[36] L. Castillo-Saavedra, N. Gebodh, M. Bikson, C. Diaz-Cruz, R. Brandao, L. Coutinho, D. Truong, A. Datta, R. Shani-Hershkovich, M. Weiss, I. Laufer, A. Reches, Z. Peremen, A. Geva, L.C. Parra, F. Fregni, Clinically effective treatment of fibromyalgia pain with high-definition transcranial direct current stimulation: phase II open-label dose optimization, J. Pain 17 (2016) 14-26, http://dx.doi.org/10.1016/j.jpain.2015.09.009.

[37] A.R. Brunoni, L. Valiengo, A. Baccaro, T.A. Zanão, J.F. de Oliveira, A. Goulart, P.S. Boggio, P.A. Lotufo, I.M. Benseñor, F. Fregni, The sertraline vs. electrical current therapy for treating depression clinical study: results from a factorial, randomized, controlled trial, JAMA Psychiatry 70 (2013) 383-391, http://dx. doi.org/10.1001/2013.jamapsychiatry.32.

[38] S. Straudi, F. Fregni, C. Martinuzzi, C. Pavarelli, S. Salvioli, N. Basaglia, tDCS and robotics on upper limb stroke rehabilitation: effect modification by stroke duration and type of stroke, Biomed Res. Int. 2016 (2016) 5068127, http://dx. doi.org/10.1155/2016/5068127.

[39] L.A. Collange Grecco, N. de Almeida Carvalho Duarte, M.E. Mendonça, M. Galli, F. Fregni, C.S. Oliveira, Effects of anodal transcranial direct current stimulation combined with virtual reality for improving gait in children with spastic diparetic cerebral palsy: a pilot, randomized, controlled, double-blind, clinical trial, Clin. Rehabil. 29 (2015) 1212-1223, http://dx.doi.org/10.1177/ 0269215514566997.

[40] M.E. Mendonca, M. Simis, L.C. Grecco, L.R. Battistella, A.F. Baptista, F. Fregni, Transcranial direct current stimulation combined with aerobic exercise to optimize analgesic responses in fibromyalgia: a randomized placebo-controlled clinical trial, Front. Hum. Neurosci. 10 (2016) 68, http:// dx.doi.org/10.3389/fnhum.2016.00068.

[41] A. Antal, M.A. Nitsche, W. Kruse, T.Z. Kincses, K.P. Hoffmann, W. Paulus, Direct current stimulation over V5 enhances visuomotor coordination by improving motion perception in humans, J. Cogn. Neurosci. 16 (2004) 521-527, http:// dx.doi.org/10.1162/089892904323057263.

[42] A. Pascual-Leone, J. Grafman, M. Hallett, Modulation of cortical motor output maps during development of implicit and explicit knowledge, Science 263 (5151) (1994) 1287-1289, http://dx.doi.org/10.1126/science.8122113, PMID: 8122113. 